\title{
Integrating Carbon Nanomaterials with Metals for Bio-sensing Applications
}

\author{
Sami Sainio ${ }^{1,2} \cdot$ Elli Leppänen $^{3} \cdot$ Elsi Mynttinen $^{3} \cdot$ Tommi Palomäki $^{3} \cdot$ Niklas Wester $^{2} \cdot$ Jarkko Etula $^{3} \cdot$ Noora Isoaho $^{3}$. \\ Emilia Peltola ${ }^{3} \cdot$ Jessica Koehne $^{4} \cdot$ M. Meyyappan ${ }^{4} \cdot$ Jari Koskinen $^{2} \cdot$ Tomi Laurila $^{3}$
}

Received: 29 August 2019 / Accepted: 29 August 2019 / Published online: 14 September 2019

(C) The Author(s) 2019

\begin{abstract}
Age structure in most developed countries is changing fast as the average lifespan is increasing significantly, calling for solutions to provide improved treatments for age-related neurological diseases and disorders. In order to address these problems, a reliable way of recording information about neurotransmitters from in vitro and in vivo applications is needed to better understand neurological diseases and disorders as well as currently used treatments. Likewise, recent developments in medicine, especially with the opioid crisis, are demanding a swift move to personalized medicine to administer patient needs rather than populationwide averages. In order to enable the so-called personalized medicine, it is necessary to be able to do measurements in vivo and in real time. These actions require sensitive and selective detection of different analytes from very demanding environments. Current state-of-the-art materials are unable to provide sensitive and selective detection of neurotransmitters as well as the required time resolution needed for drug molecules at a reasonable cost. To meet these challenges, we have utilized different metals to grow carbon nanomaterials and applied them for sensing applications showing that there are clear differences in their electrochemical properties based on the selected catalyst metal. Additionally, we have combined atomistic simulations to support optimizing materials for experiments and to gain further understanding of the atomistic level reactions between different analytes and the sensor surface. With carbon nanostructures grown from $\mathrm{Ni}$ and $\mathrm{Al}+\mathrm{Co}+\mathrm{Fe}$ hybrid, we can detect dopamine, ascorbic acid, and uric acid simultaneously. On the other hand, nanostructures grown from platinum provide a feasible platform for detection of $\mathrm{H}_{2} \mathrm{O}_{2}$ making them suitable candidates for enzymatic biosensors for detection of glutamate, for example. Tetrahedral amorphous carbon electrodes have an ability to detect morphine, paracetamol, tramadol, and $O$-desmethyltramadol. With carbon nanomaterial-based sensors, it is possible to reach metal-like properties in sensing applications using only a fraction of the metal as seed for the material growth. We have also seen that by using nanodiamonds as growth catalyst for carbon nanofibers, it is not possible to detect dopamine and ascorbic acid simultaneously, although the morphology of the resulting nanofibers is similar to the ones grown using Ni. This further indicates the importance of the metal selection for specific applications. However, Ni as a continuous layer or as separate islands does not provide adequate performance. Thus, it appears that metal nanoparticles combined with fiber-like morphology are needed for optimized sensor performance for neurotransmitter detection. This opens up a new research approach of application-specific nanomaterials, where carefully selected metals are integrated with carbon nanomaterials to match the needs of the sensing application in question.
\end{abstract}

Keywords Carbon $\cdot$ Carbon nanomaterials $\cdot$ Bio-sensing $\cdot$ Dopamine

Tomi Laurila

tomi.laurila@aalto.fi

1 Stanford Synchrotron Radiation Lightsource, SLAC National Accelerator Laboratory, Menlo Park, CA 94025, USA

2 Department of Chemistry and Materials Science, School of Chemical Technology, Aalto University, 02150 Espoo, Finland

3 Department of Electrical Engineering and Automation, School of Electrical Engineering, Aalto University, 02150 Espoo, Finland

4 Center for Nanotechnology, NASA Ames Research Center, Moffett Field, Mountain View, CA 94035, USA

\section{Introduction}

Based on both European and US sources [1, 2], more than a quarter of the population suffers from different neurological disorders. These figures are expected to increase as the population's age structure is changing with the increasing average lifespan. In addition to the above, the current opioid crisis raises questions if the population-wide averages for deciding the dosing for pain medication have been the right approach. Both problems could be solved by realization of personalized 
medicine, i.e., the ability to quickly, reliably, and affordably record how each of the patients responds to given treatment. For example, recording analgesic-related metabolites from blood would allow determining individual response to the administered medicine for each patient.

There is an abundant number of publications on the detection of neurotransmitters using different carbon nanomaterials $(\mathrm{CNM})$ and their composites, some of which are listed in Table 1 and in a review by Meyyappan [3]. However, as pointed out in a recent critical review [4], the connection between the sensing/electrochemical performance and the physicochemical properties of the sensing material has not been established unambiguously. The understanding of the effect of carbon, its functional groups $(\mathrm{O}, \mathrm{N})$, and the residing metal impurities from the fabrication process have recently gained more attention, but the scientific community has yet to determine what truly enables the electrocatalytic properties of the CNMs. In addition to the complex carbon-metal composite structures resulting from the growth process of these carbon nanostructures, their morphology is reported to have a significant role in the performance.

CNMs fabricated from different metal seed layers can result in similar micro- and nanoscale structures, but have clearly different electrocatalytic properties. Here, we show a collection of results from recent publications from our group and correlate them with new studies of CNM grown without metallic seed layer, and the same samples with additional Ni catalyst layer, with (tetrahedral amorphous carbon) ta-C + nanodiamonds (ND), with ta-C + Fe seed hybrid and with ta-C $+2 \mathrm{~nm} \mathrm{Ni}$ film. We have found that the use of Al-Co-Fe hybrid seed layer for CNM growth yields integrated structures that can detect dopamine (DA), ascorbic acid (AA), and uric acid (UA) sensitively and selectively (allowing simultaneous detection of the analytes) [5]. Further, using only Fe as seed for growing carbon nanofibers (CNF) allows us to selectively detect DA and AA, but the sensitivity is poor. Pt-grown CNFs are insensitive for DA and are passivated in just several measurement cycles. However, with both Pt- and Ni-grown CNF, we can detect $\mathrm{H}_{2} \mathrm{O}_{2}$ arising from the enzymatic reactions of glutamate [6]. From these two, the Pt version is much more sensitive towards $\mathrm{H}_{2} \mathrm{O}_{2}$ [6]. The detection of neurotransmitters and $\mathrm{H}_{2} \mathrm{O}_{2}$ is not feasible with morphologically similar $\mathrm{CNF}$ that do not have any catalytic metals in large quantities $(\geq 0.1$ at. \%).

We have utilized single-wall carbon nanotubes (SWCNT) with $\mathrm{Fe}$ seed and ta-C films with very low amount of metals $(<0.1$ at.\%) to successfully detect morphine (MO) and paracetamol (PA) [7], as well as tramadol (TR) and $O$ desmethyltramadol (ODMT) [8]. In the application of TR and ODMT using ta-C as sensor surface, the ability to detect these analytes is based on the very wide (nearly $4 \mathrm{~V}$ ) water window of ta-C. [7, 8].

Thus, to understand what is causing the electrocatalytic properties of the CNM, it is important to study the effects of (i) morphology (feature length, diameter, and size), (ii) carbon structure and orientation (crystalline/amorphous, basal/edge plane, and amount of defects), (iii) surface chemistry and functionalization, (iv) alloying, and (v) nature and abundance of metals.

It is practically impossible to understand the root causes behind the observed complex performance solely on the basis of the heavily convoluted experimental data, which cannot provide any atomistic level information about our systems. Thus, computational methods augmented by machine learning techniques are required (i) to deconvolute the experimental results into atomic level information and (ii) to aid in developing an atomic-scale quantitative microscopic model for various interactions occurring in the system. We have recently combined computational methods, experimental work, and machine learning techniques to deconvolute $\mathrm{X}$-ray absorption data into atomic-scale surface chemical information by utilizing the so-called fingerprint spectra [9-11]. This atomic-scale

Table 1 Collection of materials sensitive towards DA and capable of selective detection of DA and AA

\begin{tabular}{llcll}
\hline Electrode material & Seed material/metal catalyst & Lowest measured DA concentration (nM) & Method & Ref. \\
\hline $50 \mathrm{~nm}$ ta-C + MWCNT & $\mathrm{Al}+\mathrm{Co}+\mathrm{Fe}$ & 50 & $\mathrm{CV}$ & Palomäki et al. 2018 \\
$15 \mathrm{~nm}$ ta-C + MWCNT & $\mathrm{Al}+\mathrm{Co}+\mathrm{Fe}$ & 10 & $\mathrm{CV}$ & Palomäki et al. 2018 \\
$7 \mathrm{~nm}$ ta-C + CNT & $\mathrm{Al}+\mathrm{Co}+\mathrm{Fe}$ & 500 & $\mathrm{CV}$ & Sainio et al. 2015a \\
$7 \mathrm{~nm}$ ta-C + CNF & $\mathrm{Ni}$ & 500 & $\mathrm{CV}$ & Sainio et al. 2015b \\
$\mathrm{CNF}$ & $\mathrm{Ni}$ & 50 & DPV & Rand et al. 2013 \\
$\mathrm{CNS}$ & $\mathrm{Pt}$ & 1000 & $\mathrm{CV}$ & Wang et al. 2011 \\
GC + MWCNT & $\mathrm{Pt}$ & 5000 & $\mathrm{CV}$ & Dursun and Gelmez 2010 \\
& & 61 & DPV & Dursun and Gelmez 2010 \\
MWCNT & $\mathrm{Ta}$ & 10,000 & $\mathrm{CV}$ & Poh and Loh 2004 \\
N-MWCNT & $\mathrm{C}_{10} \mathrm{H}_{10} \mathrm{Fe}$ & 120 & $\mathrm{CV}$ & Tsierkezos et al. 2016 \\
\hline
\end{tabular}

CNS carbon nanosheet, $M W C N T$ multi-wall carbon nanotube 
chemical information can then be compared with the observed electrochemical behavior to reveal the fundamental connections between surface chemistry and electrocatalysis. This information then gives us the possibility to tailor the carbon surface chemistry for a specific application. Likewise, we have utilized density functional-based methods to investigate atomic level interactions between different catalyst metals and carbon to rationalize the different morphologies observed.

The above machine learning augmented by computational approach could also prove to be extremely useful for interpretation and rationalization of electrochemical data, since many of the recorded voltammograms contain heavily overlapping peaks. However, despite the main peaks overlapping, it is likely that if the whole voltammogram of a given analyte (its fingerprint) is "learned," features that are unique for a given analyte can be derived. Thus, by learning these fingerprints of different analyte reactions on given electrode materials, it could be possible to enable the required selectivity by machine learning-induced peak deconvolution.

\section{Experimental}

CNMs utilizing metal seed layers were grown using either chemical vapor deposition (CVD) or plasma-enhanced CVD (PECVD) process at temperatures of 550 to $750{ }^{\circ} \mathrm{C}$. The processes are explained in detail in our previous publications [12-14]. Briefly, the hybrid Al-Co-Fe seed layered material was deposited on top of a $15-$ and $50-\mathrm{nm}$ ta-C containing $\mathrm{Si}$ wafer using an electron beam [5] aiming for $0.2 \mathrm{~nm}$ of $\mathrm{Al}$, $2 \mathrm{~nm}$ of Fe, and $2 \mathrm{~nm}$ of Co. After deposition, the samples were taken to Black Magic CVD reactor (Aixtron, Germany) and heated up to $550{ }^{\circ} \mathrm{C}$ for $10 \mathrm{~min}$ with $\mathrm{NH}_{3}$ flow at $250 \mathrm{sccm}$ [12]. Samples with $20 \mathrm{~nm} \mathrm{Ni}, 20 \mathrm{~nm} \mathrm{Fe}$, and $10 \mathrm{~nm}$ Pt seed layers were deposited on top of $7 \mathrm{~nm}$ ta-C film and grown in Black Magic PECVD (Aixtron, Germany) chamber at $750{ }^{\circ} \mathrm{C}$ for $30 \mathrm{~min}$ with $\mathrm{C}_{2} \mathrm{H}_{2}$ flow of $25 \mathrm{sccm}$ and $\mathrm{NH}_{3}$ flow of $125 \mathrm{sccm}$. The ND-grown carbon nanofibers were also fabricated using the PECVD reactor mentioned above. The carboxylated nanodiamonds were deposited on the initial sample surface by drop-casting, where commercially available aqueous diamond solution with a concentration of $5 \mathrm{wt} \%$ (Vox, Carbodeon, Finland) was diluted to a concentration of $0.05 \mathrm{wt} \%$ with deionized water. A $40-\mu \mathrm{L}$ drop was then drop-casted on the sample surface, placed on a glass slide. Finally, the samples were dried on a preheated hot plate at $85^{\circ} \mathrm{C}$ for $10 \mathrm{~min}$ to accelerate the evaporation of solvent. The ND used here are commercially available detonation ND with brand name Vox by Carbodeon for CNF growth (carboxyl functionalized) and Andante (oxygen functionalized surface with non-specific groups) for ta-C + ND and for the ND + $20 \mathrm{~nm} \mathrm{Ni} \mathrm{CNF}$ growth.
Cyclic voltammetry (CV) measurements were carried out using Gamry Reference 600 or $600+$ potentiostat. A threeelectrode setup was used with an $\mathrm{Ag} / \mathrm{AgCl}$ reference electrode (Radiometer Analytica) and a Pt wire (Goodfellow) as a counter electrode. Dopamine hydrochloride, L-ascorbic acid, and uric acid were purchased from Sigma-Aldrich. Fresh solutions of DA, AA, and UA were prepared on the day of the measurements. Phosphate buffer saline (PBS) with $\mathrm{pH} 7.4$ was used as an electrolyte. All solutions were purged with nitrogen for $30 \mathrm{~min}$ before and blanketed during the experiments. All measurements were conducted at room temperature in a Faraday cage. The geometric area of the $\mathrm{Al}+\mathrm{Co}+\mathrm{Fe}$-grown electrode was $0.071 \mathrm{~cm}^{2}$ whereas for rest of the electrode area was $0.031 \mathrm{~cm}^{2}$.

High-resolution transmission electron microscopy (HRTEM) studies were carried out using JEOL JEM-2200FS under $200 \mathrm{kV}$. Samples were prepared by EAG Laboratories (USA) using focused ion beam (FIB) thinning of an extracted lamella. Scanning electron microscopy (SEM) was carried out with JEOL and Hitachi S-4700 scanning electron microscopes. Energy-dispersive X-ray spectroscopy (EDS) was carried out at low magnification using Tescan MIRA3 scanning electron microscope equipped with Thermo Scientific UltraDry silicon drift X-ray detector. Five spots approximately $1 \times 1 \mathrm{~mm}$ in size were analyzed for each sample.

\section{Results}

In the analysis and discussion of the physicochemical results from the new carbonaceous nanomaterials, we will utilize our most well-studied material as the reference, a 7-nm-thick ta-C layer deposited on 20-nm Ti adhesion layer deposited on $\mathrm{Si}$ wafer. The fabrication process, properties of the resulting film, and its electrochemical performance have been described and discussed in considerable detail in our previous publications $[5,12]$. Electrochemical performance of the ta-C films towards DA and AA is shown in Fig. 1 for reference and there is both the lack of sensitivity and selectivity when only one carbon allotrope is utilized.

Both physiologically meaningful sensitivity and selectivity must be achieved to evaluate the material's applicability for neurotransmitter detection. This means concentrations as low as 5-700 $\mathrm{nM}$ for DA $[15,16]$ and clear peak separation in the voltammogram against (at least) AA and UA (see Fig. 3d and $\mathrm{f}$ for proper peak separation). Additionally, it is necessary to use $\mathrm{CV}$ measurements instead of differential pulse voltammetry (DPV) measurements to capture real-time data.

As Fig. 1a shows, the sensitivity of the ta-C film towards DA is nearly adequate at $500 \mathrm{nM}-1 \mu \mathrm{M}$ but that the peak separation between DA and AA in panel b is too small to selectively detect them as separate peaks. 


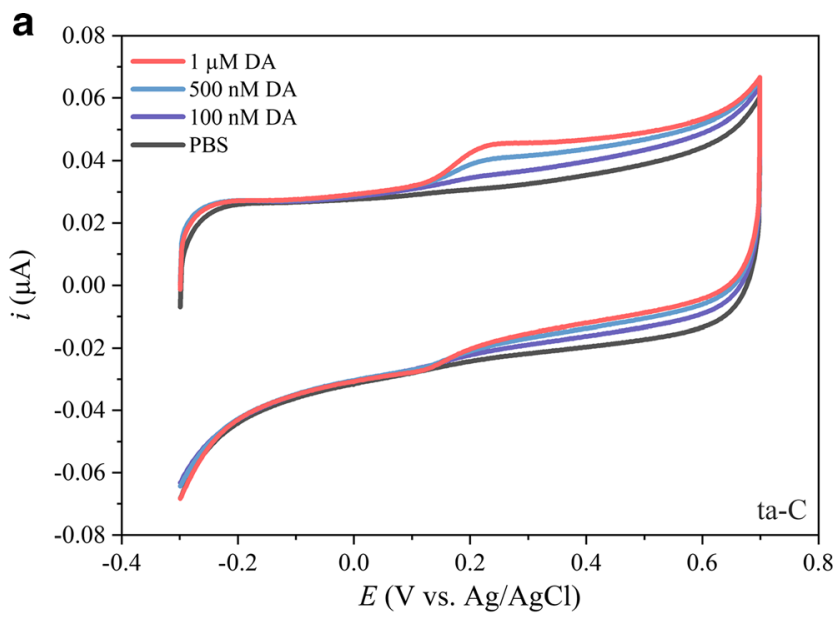

b

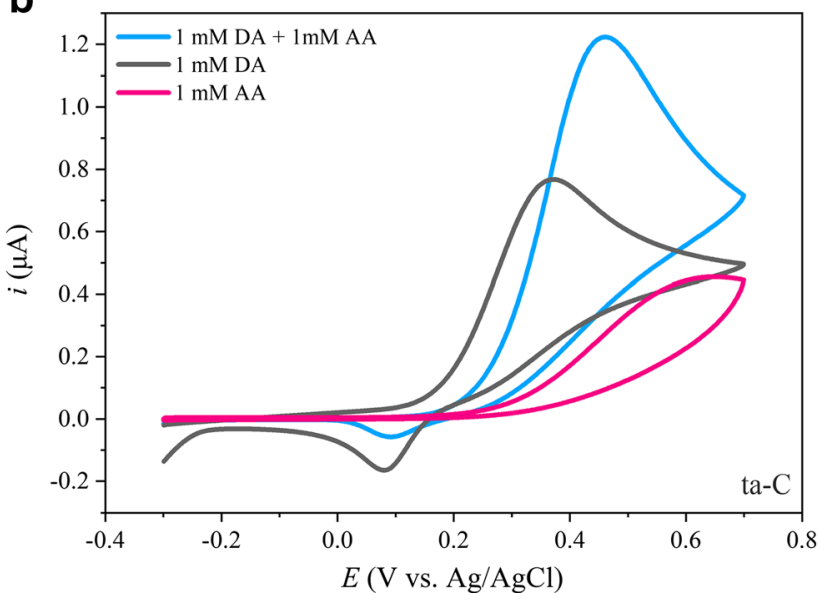

Fig. 1 Electrochemical performance of the $\mathrm{Si}+20 \mathrm{~nm} \mathrm{Ti}+7 \mathrm{~nm}$ ta-C thin film is shown in panels $\mathbf{a}$ and $\mathbf{b}$. Scan rate $50 \mathrm{mV} / \mathrm{S}$ for all measurements is shown [5]

\section{Structural Characterization}

In order to induce the required sensitivity and especially selectivity, we have utilized the so-called integrated carbon nanomaterials where different carbon allotropes are integrated together with the aid of catalyst materials, which are typically metals. We present the results from these studies below and try to find common roots that could be identified behind the increased sensitivity and selectivity. We will start by considering the metal-free carbon nanofibers where nanodiamonds have been used as a catalyst layer instead of the typical metals.

Comparing the HRTEM micrographs from the ND-grown CNF shown in Fig. 2a-c, it is evident, that the morphology of the resulting fibers is similar to earlier results $[14,17]$ where 700 -nm-long and tens to hundreds of nm wide fibers have resulted from Ni [18], Fe (as shown in SEM micrographs in Fig. 2), and Pt [19] seeds under similar PECVD conditions. The main differences are that the ND-grown fibers lack the metallic tip found from the metal seed grown fibers, as expected. The visible darker-colored grains in the ND-grown fiber walls and body are titanium that was used as an adhesion layer for the ta$\mathrm{C}$ that was deposited under the ND film. Additionally, there is a difference in the carbon forming the fibers. The ND-grown fibers seem to have mainly amorphous carbon (a-C) structure, the Ni, Fe, and Pt-grown fibers have more clear ordered structures, but even the Ni and Pt fibers are different in their structure which will be discussed in more detail later.

\section{Electrochemical Detection of Neurotransmitters}

Electrochemical measurements for the metal-free ND-grown $\mathrm{CNF}, \mathrm{Fe}$-grown $\mathrm{CNF}$, and $\mathrm{Al}+\mathrm{Co}+\mathrm{Fe}$-grown $\mathrm{CNM}$ are shown in Fig. 3 a and b, c and d, and e and f, respectively. Figure 3 clearly shows that neither the required sensitivity or selectivity is achieved with ND-grown CNF and only selectivity is achieved with Fe-grown CNF. This indicates that NDgrown $\mathrm{CNF}$, although having very similar morphology as for example the Ni-grown CNF, is not viable for neurotransmitter detection. It should be noted, that although both fiber macroscopic structure appears to be similar, fiber-like, but microscopic structure differs as the ND-grown CNF are mainly a-C, whereas the Ni-grown $\mathrm{CNF}$ are crystalline. Interestingly, both sensitivity and selectivity over DA for both AA and UA were achieved with $\mathrm{Al}+\mathrm{Co}+\mathrm{Fe}$-grown $\mathrm{CNM}$. This is proposed to arise from a mixture of properties: (i) there are at least $\mathrm{Fe}$ and Co metal nanoparticles available, (ii) there are crystalline carbon areas available, and (iii) the morphology in both microand macroscale is optimal for electrocatalysis (high surface area and the CNM forms a porous network that enables enrichment of the analyte).

The only material that we have found to show selective behavior towards DA and AA without growth seed metal particles is ta-C + partially reduced graphene oxide (PRGO) hybrid electrode that has been treated with concentrated nitric acid before the measurements [20]. Based on the X-ray photoelectron spectroscopy (XPS) studies, the surface of the PRGO is covered by more than 30 at.\% oxygen, more than 10 at.\% Si, and traces of at least $\mathrm{S}, \mathrm{N}, \mathrm{B}, \mathrm{Ca}$, and possible traces of $\mathrm{Al}, \mathrm{F}, \mathrm{Mn}$, and $\mathrm{Na}$ (it should be noted that $\mathrm{KMnO}_{4}$ is used in the process of making the PRGO and the Mn is not removed from the material by any specific process and is likely present in higher concentration in the bulk of the PRGO). Due to the complexity of the PRGO surface, it is hard to attribute the selective behavior to any single component of the system. However, we believe that the enhanced performance is due to the heavily oxidized system, increased surface area due to the formation of a porous membrane-like structure, and to the Mn present in the system.

Table 1 shows the results for different materials' capability for DA and AA separation, the electrochemical method used, and the seed material used for the CNM growth. Only materials with capabilities for simultaneous DA and AA detection are shown. 

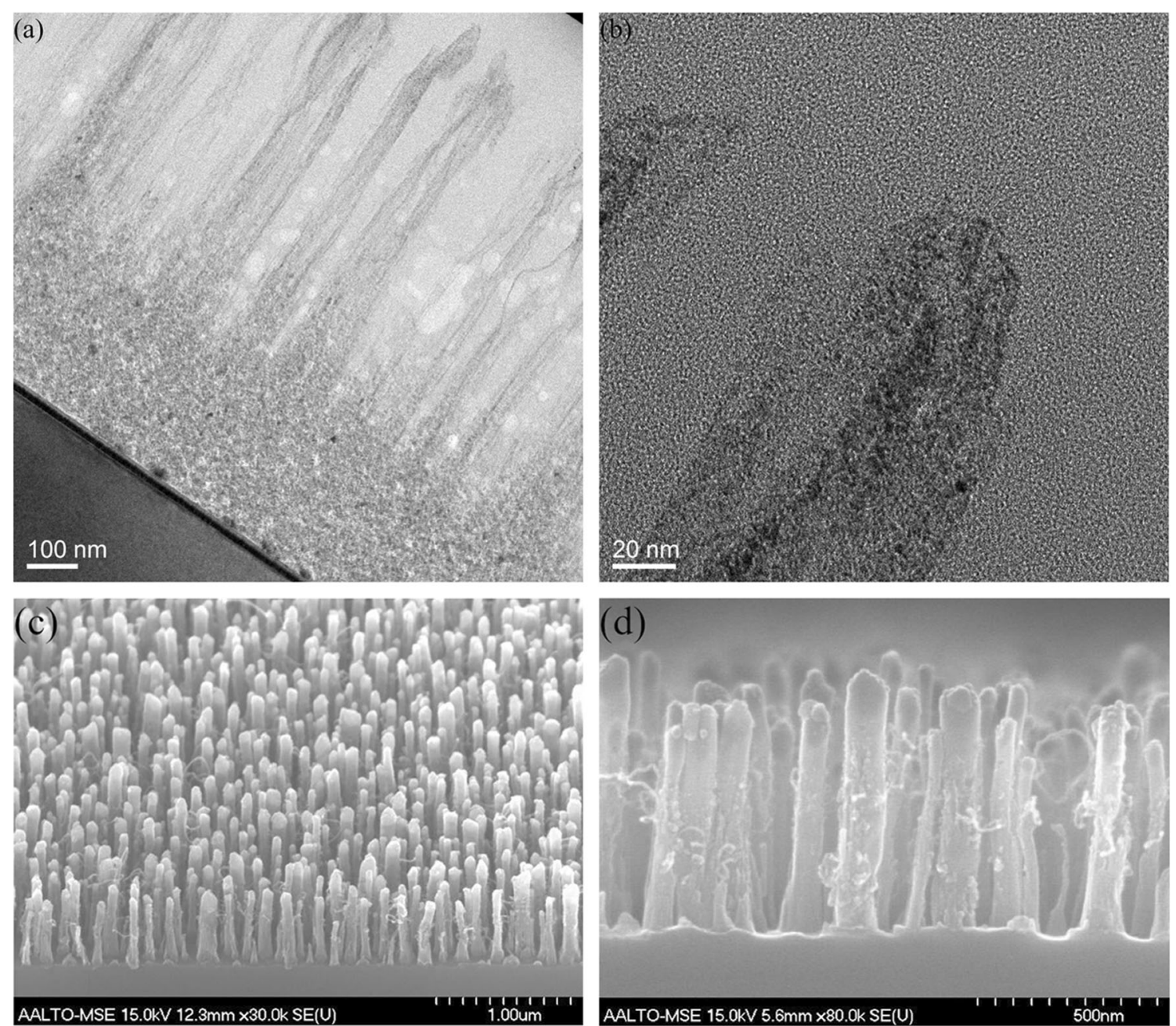

Fig. 2 a, b TEM micrographs of ND-grown CNF where in panel a, the 700-nm-tall fibers are grown on top of a ND layer, in panel $\mathbf{b}$, the tip of the resulting fiber is shown and in panel $\mathbf{c}$, body of the fiber is shown that

As shown in Table 1, the only materials we have found to fulfill the requirements of sensitivity and selectivity are the hybrid $\mathrm{Fe}+\mathrm{Co}+\mathrm{Al}$-grown $\mathrm{CNM}$ and the Ni-grown $\mathrm{CNF}$. Other results listed in Table 1 show that simultaneous detection of DA and AA is possible with CNM grown from $\mathrm{Pt}$, Ta, and $\mathrm{Fe}$. Based on our knowledge, the $\mathrm{Fe}+\mathrm{Co}+\mathrm{Al}$-grown $\mathrm{CNM}$ is the only material capable of detecting DA, AA, and UA sensitively and selectively with CV [5], whereas the Nigrown CNF has shown adequate performance only with DPV [21]. Results with DPV are not relevant due to the lack of application required time resolution (in the case of neurotransmitter detection).

Some materials, such as the CNF grown using Fe seed, show clear peak separation for DA and AA (see Fig. 3) but lack the necessary sensitivity. Other materials without metal particles, like the ta-C films, show good enough sensitivity but lack the selectivity. Furthermore, by building carbon hybrid nanomaterials, e.g., by combining ta-C and ND, detection of concentrations down to $100 \mathrm{nM} \mathrm{DA}$ is possible. Also, by

shows amorphous areas where the diamonds are scattered around the body of the fiber. c, d SEM micrographs of the Fe-grown CNF showing similar morphology as the fibers in refs [17-19]

utilizing surface oxidizing treatments such as nitric acid treatments [18], improvements in the sensitivity have been achieved [5, 7, 20, 22]. Despite all these efforts, the ability to selectively detect DA and AA has not been reached using only metal-free (or very low metal concentration) carbonbased nanomaterials.

As shown earlier, the Ni-grown CNFs are both sensitive for DA and selective between DA and AA [13]. To study the effects of metal and carbon systems in more detail, we introduced an additional 20-nm layer of $\mathrm{Ni}$ on top of a ta-C $+\mathrm{ND}$ surface before the growth process. The resulting CNF shows selectivity for DA over AA, but the sensitivity for DA was still only $10 \mu \mathrm{M}$. This could be related to the fiber structure as its sidewalls, the base, and the tip were different than those for CNF grown without the ND layer (see Fig. 5 in the "Discussion" section for illustration of the different fiber morphologies). Further, we tested deposition of a $2 \mathrm{~nm}$ (nonuniform coating) of Ni on top of a 7-nm ta-C. The selectivity for DA and AA was not achieved with this configuration. 


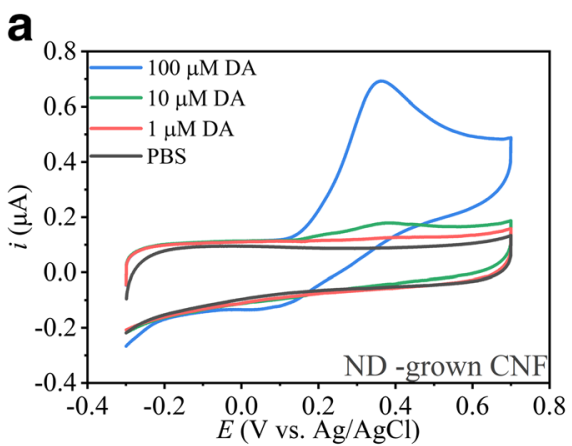

b

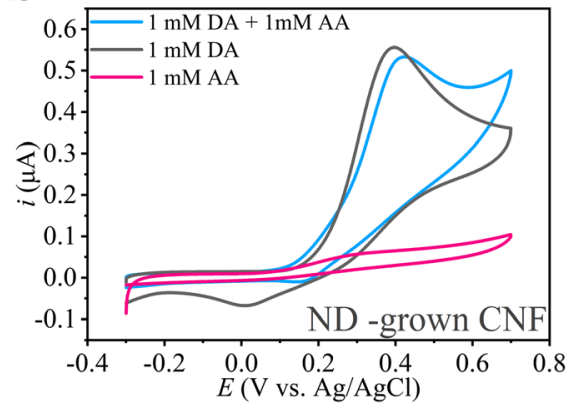

C

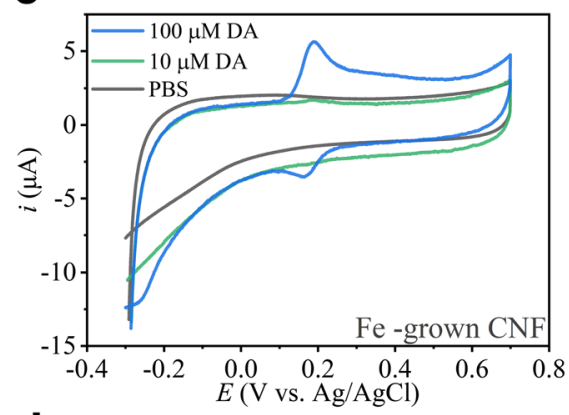

d

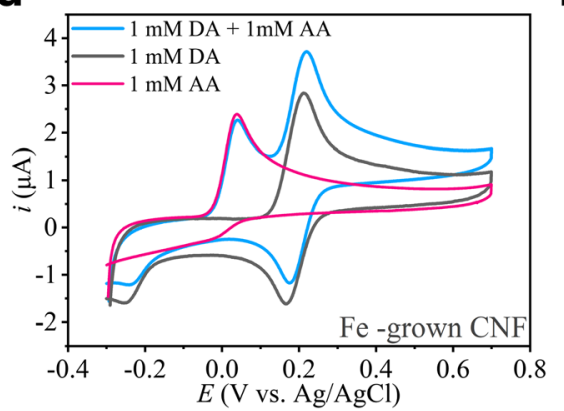

e

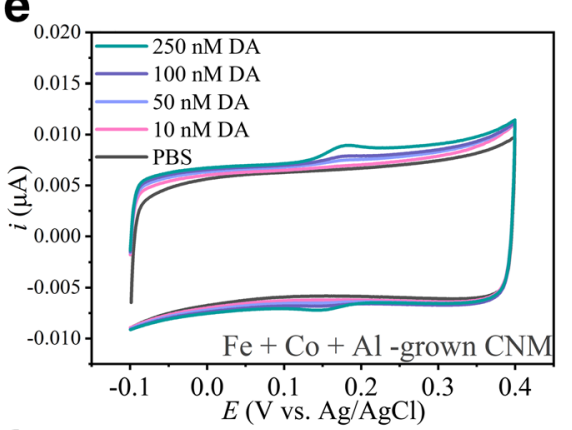

f

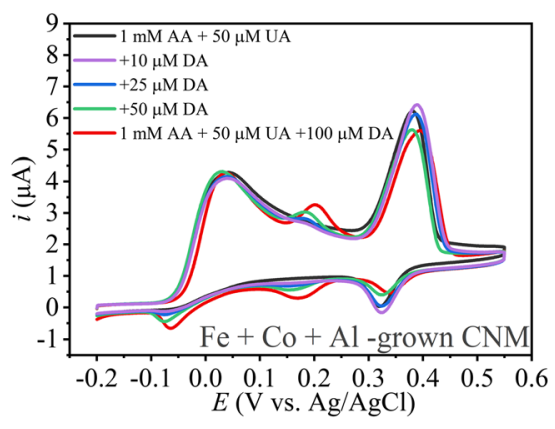

Fig. 3 Cyclic voltammograms from measurements with $\mathrm{ND}, \mathrm{Fe}$, and $\mathrm{Fe}+$ $\mathrm{Co}+\mathrm{Al}$-grown CNM. In panels $\mathbf{a}$ and $\mathbf{b}$, the ND-grown CNF shows no detection for AA and low sensitivity towards DA. In panels $\mathbf{c}$ and $\mathbf{d}, \mathrm{Fe}-$ grown CNF show selectivity of DA and AA but low sensitivity to DA and in panels $\mathbf{e}$ and $\mathbf{f}$, the best results so far for both sensitivity and selectivity are shown with $\mathrm{Fe}+\mathrm{Co}+\mathrm{Al}$-grown $\mathrm{CNM}$. Scan rate of $50 \mathrm{mV} / \mathrm{s}$ is shown for all measurements

\section{Electrochemical Detection of $\mathbf{H}_{\mathbf{2}} \mathbf{O}_{2}$}

Ni particles on the surface did not seem to improve selectivity. Additionally, introducing the $\mathrm{Ni}$ on top of the ta-C decreased the sensitivity of the material. Furthermore, a sample with ND deposited on top of the ta-C without growth was measured to inspect its performance. The sample exhibited no selectivity but was sensitive to low concentrations of DA (see Fig. 3e below). These results indicate that the micro- and nanoscale morphology of the CNM is important and the placement of the metal seed particle in the CNM matrix is also relevant. Results from selectivity and sensitivity measurements for the materials listed above are shown in Fig. 4.

To improve the readability and comparability of the results along with laying a clear foundation for the upcoming discussion, we have summarized the results for the studied CNM in Table 2. Table 2 also presents the EDS results from the materials used in this study to confirm the presence of the used catalyst metals (and the Ti adhesion layer used below the ta-C). Correlation of specific amounts of these metals is not discussed in detail as we only know the bulk composition of the materials from the EDS studies. In order to understand the electrocatalytic properties of the materials, both the bulk (such as EDS and fluorescence) and surface characterization (such as Xray photoelectron spectroscopy and X-ray absorption spectroscopy) should be carried out. The catalyst metal reported in Table 2 has been confirmed using EDS with SEM and/or TEM from the final structure.
We have aimed our studies also towards the detection of glutamate (Glu). However, Glu is electrochemically inactive and it is necessary to use enzymes, typically glutamate oxidase (GluOx), and measure the product of the enzymatic reaction. For GluOx, the reaction with Glu in the presence of $\mathrm{O}_{2}$ produces $\mathrm{H}_{2} \mathrm{O}_{2}$, which can be detected electrochemically. Thus, materials used for glutamate sensor applications need to fulfill the following criteria: (i) they have to provide a suitable platform for GluOx immobilization and (ii) they have to exhibit good properties for detecting $\mathrm{H}_{2} \mathrm{O}_{2}$. So far, we have inspected $\mathrm{H}_{2} \mathrm{O}_{2}$ detection on several materials including ta- $\mathrm{C}$, ta-C with $\mathrm{Pt}$ alloying, ND-grown CNFs, Ni-grown CNFs, and Pt-grown CNFs [6, 19, 23-25]. The best results from these measurements were achieved by using Pt-grown CNFs. We have attributed this in particular for the capability of detecting $\mathrm{H}_{2} \mathrm{O}_{2}$ with the highly catalytic Pt particles at the tip and sidewalls of the fibers. Moreover, we have shown that it is possible to immobilize GluOx on both Ni- and Pt-grown CNFs by covalently crosssidewalls and detect Glu with these biosensors [6, 24].

\section{Electrochemical Detection of Analgesics}

We have recently shown that detection of PA and MO simultaneously is possible with planar tetrahedral amorphous carbon (ta-C) thin film electrode if its Ti adhesion layer is partially linking the enzyme on the carboxylic groups present in the fiber 
a

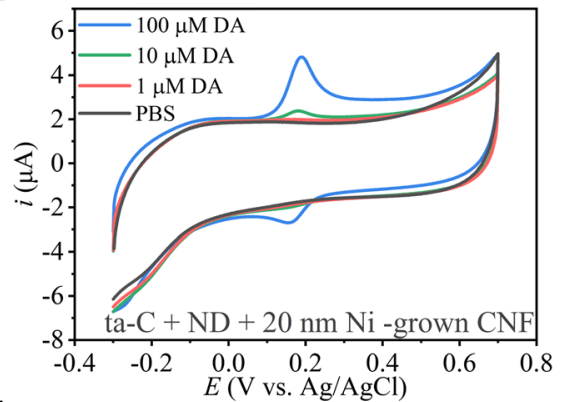

b

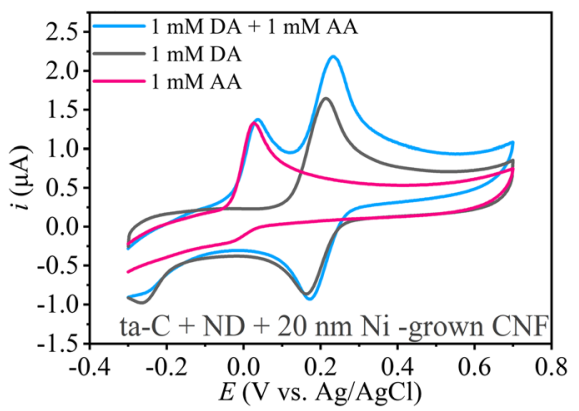

C

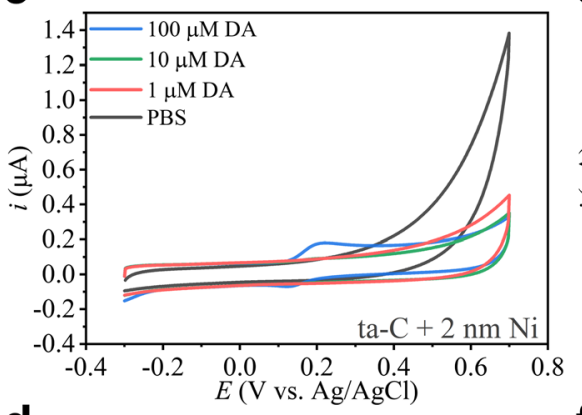

d

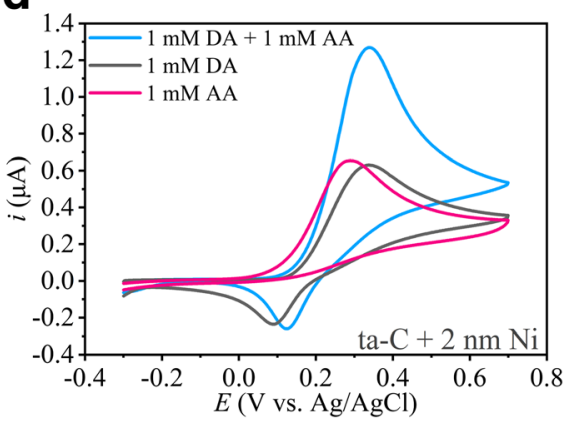

e

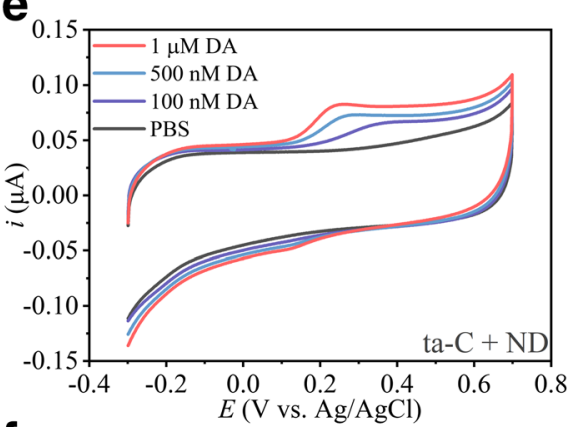

f

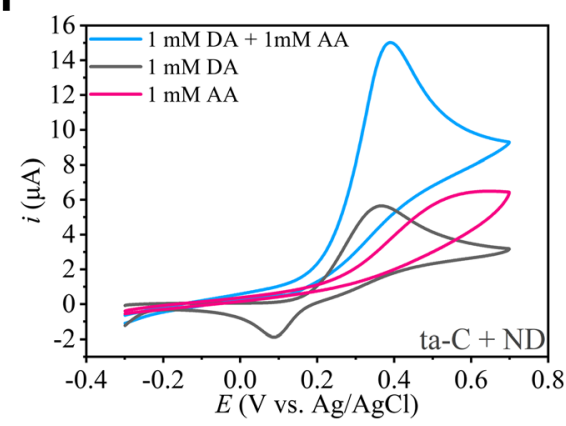

Fig. 4 ta-C + ND + $20 \mathrm{~nm} \mathrm{Ni-grown} \mathrm{CNF}(\mathbf{a}, \mathbf{b})$ shows clear selectivity between DA and AA, but only $10-\mu \mathrm{M}$ detection limit for DA. ta-C $+2 \mathrm{~nm}$ $\mathrm{Ni}$ in panels $\mathbf{c}$ and $\mathbf{d}$ show no selectivity and $100-\mu \mathrm{M}$ detection limit for

exposed [7]. Selective detection of PA and MO is not achieved without exposing the $\mathrm{Ti}$ and is not possible with just plain Ti.

On the other hand, detecting TR and ODMT sensitively and selectively is only enabled by using ta-C thin films without the underlying $\mathrm{Ti}$. The simultaneous detection is, for the most part, enabled by the large water window of ta-C, allowing separate signals for these two analytes within the measurement window. If a $\mathrm{Ti}$ layer is added below the ta- $\mathrm{C}$ film, the water window is decreased to an extent where the signal for ODMT is buried under the oxygen evolution. These two examples again emphasize the interplay between chemistry and morphology.

\section{Discussion}

There are several variables that do change between the given CNM that radically change their electrochemical behavior. We can establish the factors that affect the performance include at least (i) the selected metal present in the carbon material, such as the catalyst particles or partly exposed metal sublayer in planar carbon matrix [7]; (ii) the morphology of the surface (porous spaghetti-like, forest-like, planar, fiber width and length and the size of the catalyst particle); and (iii) the nanoscale orientation of the carbon at the surface of the electrode (crystalline or amorphous and the orientation of the graphene planes) and the oxidation state of the carbon and the metal particles on the material surface.
DA. In panels e and $\mathbf{f}$, the ta-C $+\mathrm{ND}$ results are shown that show adequate sensitivity but poor selectivity. Scan rate $50 \mathrm{mV} / \mathrm{S}$ for all measurements is shown

Best results that we have achieved towards neurotransmitter detection are with materials that have (i) the metal seed particles or (ii) are heavily oxidized (and possibly other factors related to listed impurities as stated earlier with the PRGO results) and (iii) have porous-like membrane structures (as the PRGO-layer or CNT layer) that possibly leads to thin liquid film formation that, in turn, promotes adsorption. For $\mathrm{H}_{2} \mathrm{O}_{2}$ detection, we have seen the best results with Pt-grown $\mathrm{CNF}$, which is not surprising owing to the excellent catalytic properties of $\mathrm{Pt}$ towards $\mathrm{H}_{2} \mathrm{O}_{2}$. In the detection of $\mathrm{MO}$ and $\mathrm{PA}$, the exposure of metal particles is similarly crucial for the separation of the two signals. Contrarily, with TR and ODMT, simultaneous detection is achieved with a ta-C film without an underlying Ti layer and thus without any exposed metal particles [8]. The detection of these was not achieved with Fe-containing SWCNTs.

Looking back to the earlier results, the following obvious conclusion arises: every target application has its own requirements and characteristics and the sensing materials used for one is unlikely to be the best for the other. Thus, understanding the connections between the surface chemistry, nanoscale morphology, and the electrocatalytic behavior is the key to enable fabrication of application-specific CNMs.

For DA, AA, and UA, the best material so far for simultaneous and sensitive detection is the CNM resulting from CVD growth with hybrid $\mathrm{Fe}+\mathrm{Co}+\mathrm{Al}$ seed at low temperature. This material has multi-metal seed particles and a defective porous network of CNM at the surface (see Fig. 5). Similar 

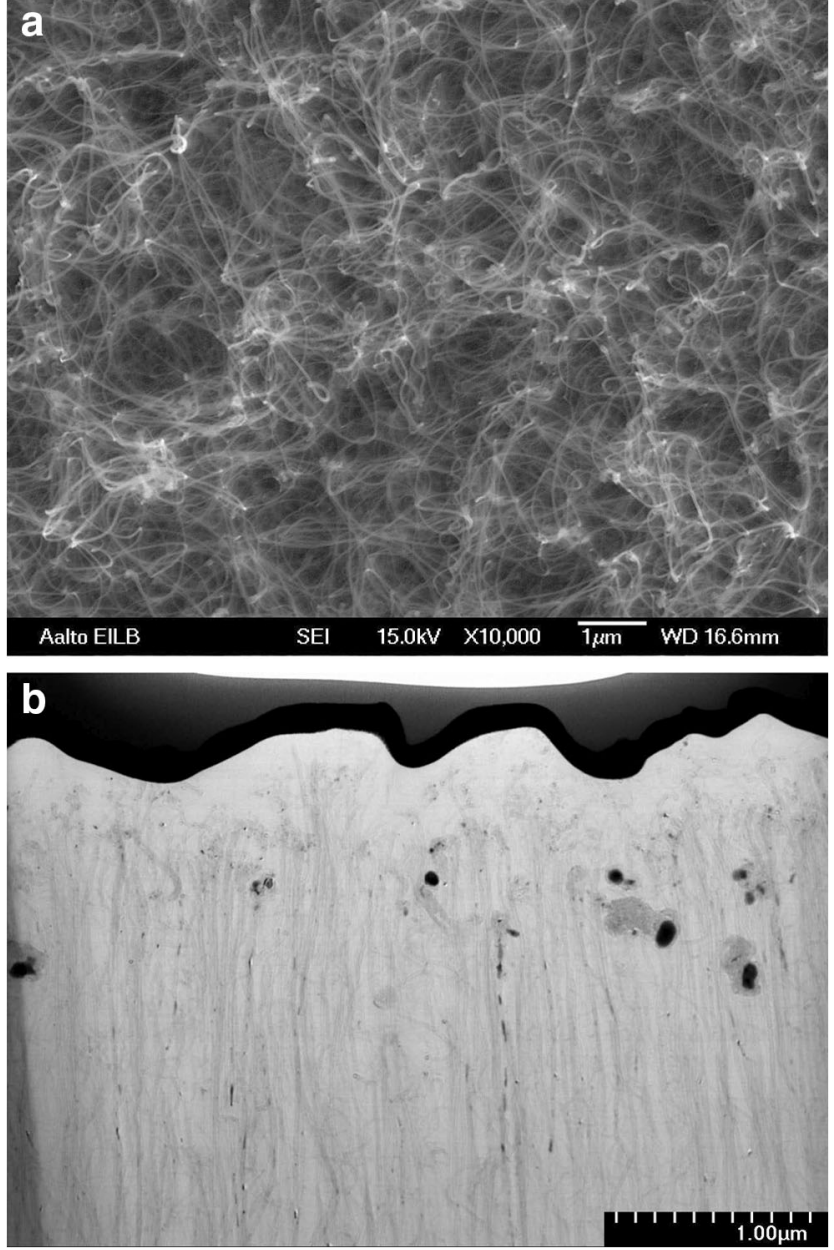

Fig. 5 SEM (a) and TEM (b) micrographs from hybrid CNM with multimetal seed showing the porous network in panel a and the varying sizes and availability of the metal seed particles in panel $\mathbf{b}$

performance was reached with Ni-grown CNF; however, selectivity was achieved only via DPV [21].

CNFs that show selectivity for DA and AA were grown from both $\mathrm{Ni}$ and Fe catalysts, but the latter had too low sensitivity towards DA. Interestingly, the same low sensitivity is also observed for CNFs grown from ND + $20 \mathrm{~nm}$ Ni seed layer. Likewise, Ni particles deposited on top of ta-C film showed no selectivity and low sensitivity. This is interesting as it seems that the Ni alone will not solve the selectivity and sensitivity issues as one could falsely conclude from comparing the results obtained with CNF grown from Ni seed and those obtained with ND-grown CNF. Thus, also the intrinsic "nanolevel" morphology of the CNF must be taken into account.

Another aspect may be the size of the nanoparticles residing mainly at the fiber tips. An example is the case with ta-C + $2 \mathrm{~nm}$ Ni deposited on top of it. There, the Ni particle size is expected to be small (diameter of just several $\mathrm{nm}$ ) compared to the variety of particle sizes available in the Ni-grown CNFs which ranges from tens of nanometers in diameter to at least $200 \times 400 \mathrm{~nm}$ particles [14] (see Fig. 6 for the graphical illustration of the fibers). We have shown that the Ni particles at the fiber tips are oxidized (although not completely) already after the growth process and can be further oxidized at least by acid treatments [18]. Thus, it is expected that polarizing them in electrochemical cell allows subsequent oxidation and reduction of the metal particles promoting electrocatalysis. Further, it is possible that smaller Ni particles would have different electrocatalytic properties than their larger counterparts owing to the more extensive oxidation, for instance. Based on the ta-C + ND $+20 \mathrm{~nm}$ Ni-grown CNF, it seems that they have the selectivity as the ta- $\mathrm{C}+\mathrm{Ni}$-grown fibers but lack the sensitivity. Here, we expect that the lack of sensitivity is related to the micro- and nanoscale morphology of the fiber. With platelet-type fibers (see references [14, 17, 18], for discussion of the fiber structures), there are edge planes of graphene sheets pointing outwards of the fiber center. This is the case also with other very sensitive fibers [21]. As discussed in the above two references, the sidewalls of the graphene point outside of the fiber center in all cases. Commonalities for both include the following: (i) their structure is crystalline and not amorphous, (ii) there is a plethora of large (tens to hundreds of nanometers in diameter) Ni particles at the tips of the fibers, and (iii) the fibers are protruding from the surface in a forest-like manner.

We anticipate that after further investigation, the ta-C + ND $+20 \mathrm{~nm}$ Ni-grown fibers will result in structures of amorphous nature either due to mixing of the ND in the growth process or due to the Ti film reacting differently to the growth process when the ND layer is present and resulting in mixing into the fiber body. The presence of the crystalline ND at the fiber surface could explain why the sensitivity of the ta-C + $\mathrm{ND}$ is better than the ta- $\mathrm{C}$ electrode alone owing to the increased active area due to roughness.

Figure 6 illustrates the different morphologies of the grown $\mathrm{CNF}$ structures and shows the graphical representation of the $\mathrm{Ti}$ layer mixing it to the fiber bodies in the ND- and Pt-grown CNF.

In our studies, the Pt-grown CNF foul very quickly (in a matter of few cycles) when used for DA measurements, but are capable of detecting $\mathrm{H}_{2} \mathrm{O}_{2}$ in low concentrations, where the Ni-grown CNF can detect $\mathrm{H}_{2} \mathrm{O}_{2}$ but are less sensitive towards it, and finally, the ND-grown CNFs are the worst of the three for the $\mathrm{H}_{2} \mathrm{O}_{2}$ detection. In comparison, the $\mathrm{Fe}+\mathrm{Co}+$ $\mathrm{Al}$-grown $\mathrm{CNM}$ seems resistant to fouling and the $\mathrm{Ni}$-grown CNF has been found to be resistant as well.

To further improve the performance of the material, as shown earlier, we can enrich the analytes in question by introducing a defective CNM that forms a porous network near the surface.

Interestingly, the ND-grown CNFs are not sensitive towards AA at all (as seen from Fig. 3b). The exact reason for this remains currently unknown. We anticipate that the Ti underlayer, which has been exposed and partially mixed to the fiber body, has a role in the inability to detect AA. If this is truly the case, it is possible that 


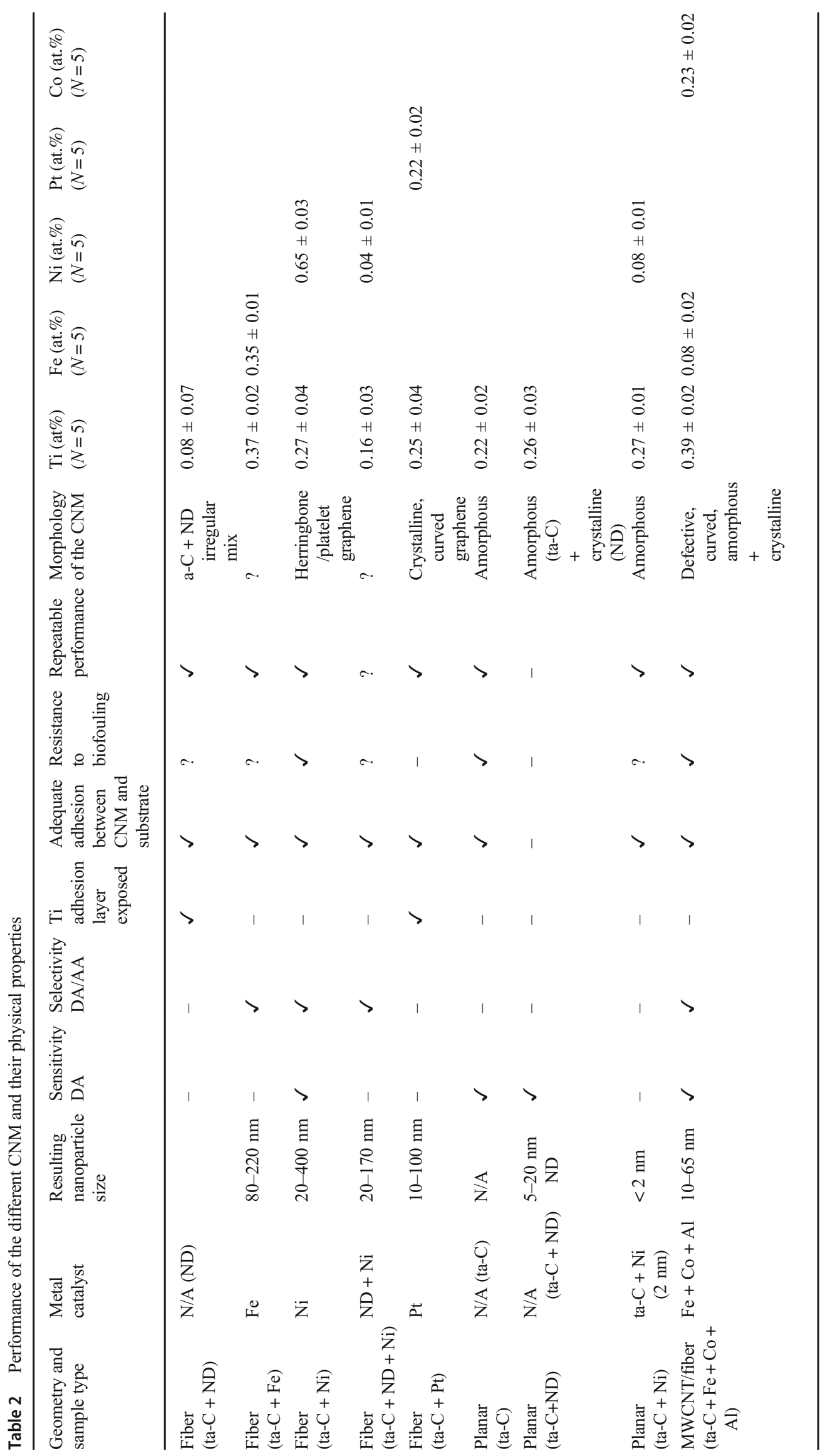


Fig. 6 Graphical illustration of the different $\mathrm{CNM}$ morphologies

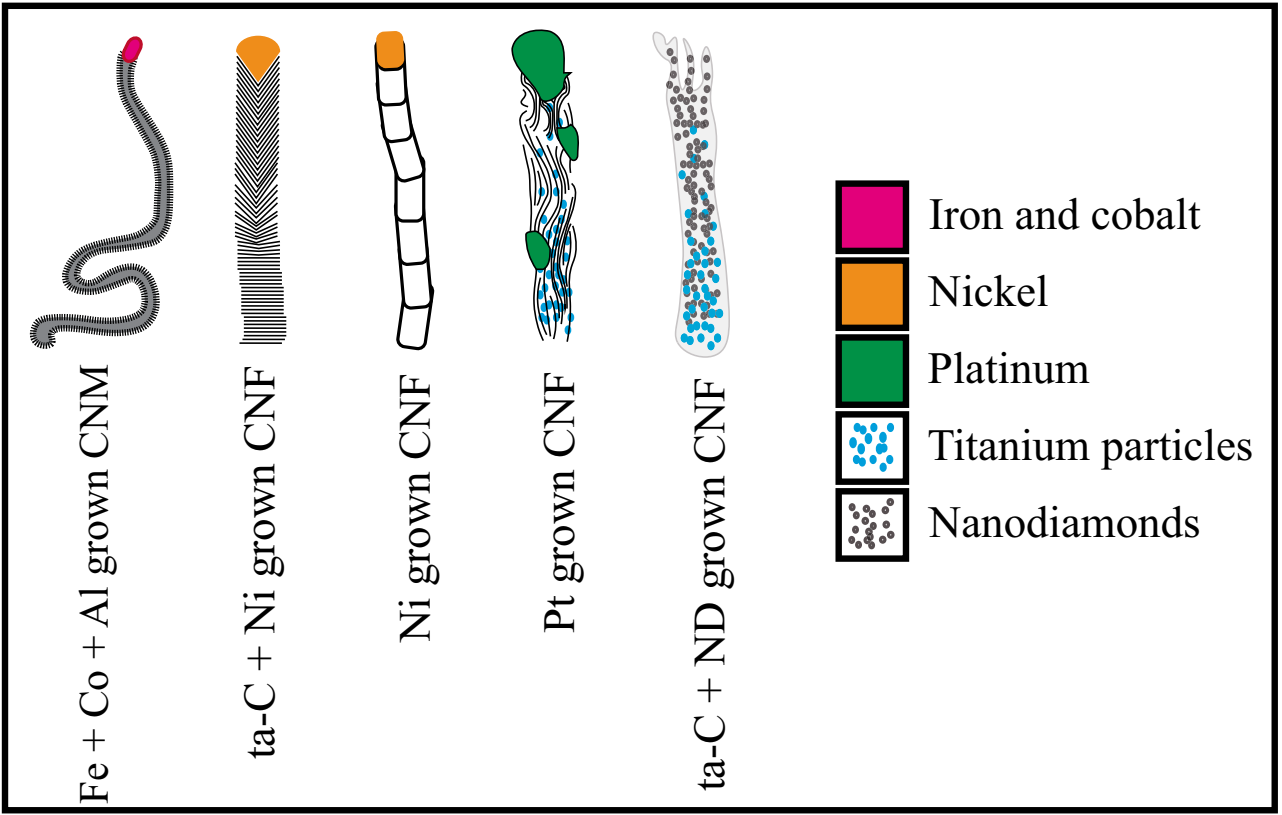

mixing Ti to the common CNM CVD process would allow selective filtering of AA. However, more studies need to be done to prove such hypothesis. These further studies will also include the use of the ND-grown CNF towards detection of PA and $\mathrm{MO}$ as the ta-C with the Ti underlayer was capable of detecting them selectively. We envision that the reasons for the inability of AA detection are at least partially related to negative surface charge of $\mathrm{TiO}_{x}$ (the Ti layer is expected to be completely covered by an oxide) and the ND. This could be true especially with the Vox brand name diamonds, which are sold having a negative zeta potential, if any of the surface functional groups stay intact through the PECVD process. In the cases where $\mathrm{Ti}$ is exposed and/or the ND negative zeta potential is making the surface charge of the structure to be negative, there could be an electrostatic repulsion between the CNM and AA (which is negatively charged).

As discussed above, the use of computational methods augmented with machine learning methods can provide us atomic level understanding of the various interactions occurring in the system under investigation and thus provide a tool to tailor surfaces for specific applications. After deconvoluting the experimental XAS spectra, for instance, into fingerprints of various functional groups, we can use this information in our adsorption calculations to mimic the real surface and obtain consequently quantitative understanding of the most feasible functional groups needed for optimal interaction between our target molecule and the electrode surface. We can also use computational screening to obtain information about the most promising catalyst metals for certain types of carbonaceous nanostructures. Although many modern techniques, such as in situ electrochemical STM and AFM, provide us with almost atomic resolution data, its interpretation requires microscopic models, which can be provided only by computational methods. This work is currently underway in our laboratory and several key results in understanding carbon surface chemistry have been achieved $[10,11$, 26-28].

\section{Conclusions}

Understanding the root causes of electrocatalytic performance of the carbon-based materials enables their applicationspecific tailoring. Through machine learning accelerated processing and simulation, a significant amount of time can be cut from the traditional trial-and-error approach in developing novel materials for sensing applications.

Results of our work show that best sensitivity and selectivity towards neurotransmitter DA and its relevant interferents AA and UA was achieved by cyclic voltammetry using hybrid CNM grown from multi-metal seed consisting of $\mathrm{Co}, \mathrm{Fe}$, and Al. This material combines the key elements seemingly required for achieving both high sensitivity and selectivity which are (i) active metal catalyst particles present in the CNM structure, (ii) CNM surface has crystalline areas available (basal plane with plenty of defects), and (iii) surface morphology of the material provides increased surface area by forming a porous network possibly resulting into the formation of thin liquid layer near the surface. There are likely numerous other metalcarbon combinations that can achieve similar performance and combining computational methods with the empirical data offers a promising tool for finding them.

We demonstrated that sensitivity towards DA can be achieved with planar, unmodified ta-C surface, and further 
improved by introducing ND on the surface. These planar electrodes are, however, not selective towards DA and AA. It seems that metal catalyst $(\mathrm{Fe}, \mathrm{Ni}, \mathrm{Fe}+\mathrm{Co}+\mathrm{Al})$ at the tips of the CNM or other non-carbon species in the structure (in the PRGO case heavy surface functionalization and/or presence of other elements) are required for selectivity. However, the metal particles alone are not enough to enable the required selectivity. The particles need to be (i) positioned at the tips, sidewalls, or mixed in the CNM matrix; (ii) of certain type(s) for DA detection and DA-AA separation (i.e., $\mathrm{Ni}$ or $\mathrm{Fe}+\mathrm{Co}+$ $\mathrm{Al}$ ); and (iii) of right size range.

On the other hand, for $\mathrm{H}_{2} \mathrm{O}_{2}$ detection, we found out that the best results were achieved using Pt-grown $\mathrm{CNF}$, whereas $\mathrm{Ni}$ and ND-grown CNF can also be used but produce lessviable results. With the Pt-grown CNFs, it is possible to detect $\mathrm{H}_{2} \mathrm{O}_{2}$ at a resolution comparable to pure Pt electrodes with just a fraction of the metal needed. Another advantage of the CNFs over bulk Pt electrodes is the possibility to covalently bind enzymes on them via cross-linking.

For the detection of analgesics, the conclusions are less straightforward. In the case of PA and MO, the best results have been achieved by a ta-C electrode with a Ti underlayer partially exposed. On the other hand, with TR and ODMT, selective detection was enabled by the wide water window of a ta-C electrode with no exposed metal particles.

To conclude, it is evident that the metal particles have a definite role in determining the electrocatalytic properties of the sensing material. It is extremely important to understand how the different metals affect the detection of certain analytes. This could further enable the selection of the desired properties for the given application based on the carbon matrix and morphology. Finally, we emphasize that supporting experimental work with computational methods paired with machine learning offers very fruitful avenues towards solving the unresolved enigma of electrocatalysis.

Acknowledgments Author S.S. acknowledges Instrumentarium Science Foundation funding. Carbodeon is acknowledged for providing the ND used in this study.

Funding Information Open access funding provided by Aalto University.

Open Access This article is distributed under the terms of the Creative Commons Attribution 4.0 International License (http:// creativecommons.org/licenses/by/4.0/), which permits unrestricted use, distribution, and reproduction in any medium, provided you give appropriate credit to the original author(s) and the source, provide a link to the Creative Commons license, and indicate if changes were made.

\section{References}

1. Gustavsson A, Svensson M, Jacobi F, Allgulander C, Alonso J, Beghi E, Dodel R, Ekman M et al (2011) Cost of disorders of the brain in Europe 2010. Eur Neuropsychopharmacol 21:718-779. https://doi.org/10.1016/j.euroneuro.2011.08.008

2. Murray CJL, Atkinson C, Bhalla K, Birbeck G, Burstein R, Chou D, Dellavalle R, Danaei G et al (2013) The state of US health, 19902010: burden of diseases, injuries, and risk factors. JAMA 310 : 591-608. https://doi.org/10.1001/jama.2013.13805

3. Meyyappan M (2015) Nano biosensors for neurochemical monitoring. Nano Convergence 2:18. https://doi.org/10.1186/s40580-015-0049-3

4. Laurila T, Sainio S, Caro MA (2017) Hybrid carbon based nanomaterials for electrochemical detection of biomolecules. Prog Mater Sci 88:499-594. https://doi.org/10.1016/j.pmatsci.2017.04.012

5. Palomäki T, Peltola E, Sainio S, Wester N, Pitkänen O, Kordas K, Koskinen J, Laurila T (2019) Corrigendum to "Unmodified and multi-walled carbon nanotube modified tetrahedral amorphous carbon (ta-C) films as in vivo sensor materials for sensitive and selective detection of dopamine" [Bios. Bioelectron. 118 (2018) 23-30]. Biosens Bioelectron 123:281-284. https://doi.org/10.1016/j.bios. 2018.08.053

6. Isoaho N, Peltola E, Sainio S, Koskinen J, Laurila T (2018) Ptgrown carbon nanofibers for enzymatic glutamate biosensors and assessment of their biocompatibility. RSC Adv 8:35802-35812. https://doi.org/10.1039/C8RA07766E

7. Wester N, Etula J, Lilius T, Sainio S, Laurila T, Koskinen J (2018) Selective detection of morphine in the presence of paracetamol with anodically pretreated dual layer Ti/tetrahedral amorphous carbon electrodes. Electrochem Commun 86:166-170. https://doi.org/10. 1016/j.elecom.2017.12.014

8. Mynttinen E, Wester N, Lilius T, Kalso E, Koskinen J, Laurila T (2019) Simultaneous electrochemical detection of tramadol and Odesmethyltramadol with Nafion-coated tetrahedral amorphous carbon electrode. Electrochim Acta 295:347-353. https://doi.org/10. 1016/j.electacta.2018.10.148

9. Sainio S, Nordlund D, Caro MA, Gandhiraman R, Koehne J, Wester N, Koskinen J, Meyyappan M et al (2016) Correlation between sp3-to-sp2 ratio and surface oxygen functionalities in tetrahedral amorphous carbon (ta-C) thin film electrodes and implications of their electrochemical properties. J Phys Chem C 120:82988304

10. Aarva A, Deringer VL, Sainio S, Laurila T, Caro MA (n.d.) Understanding X-ray spectroscopy of carbonaceous materials by combining experiments, density functional theory and machine learning. Part I: fingerprint spectra

11. Aarva A, Deringer VL, Sainio S, Laurila T, Caro MA (n.d.) Understanding X-ray spectroscopy of carbonaceous materials by combining experiments, density functional theory and machine learning. Part II: quantitative fitting of spectra

12. Sainio S, Palomäki T, Rhode $S$, Kauppila M, Pitkänen O, Selkälä T, Toth G, Moram M et al (2015) Carbon nanotube (CNT) forest grown on diamond-like carbon (DLC) thin films significantly improves electrochemical sensitivity and selectivity towards dopamine. Sens Actuators B Chem 211:177-186. https://doi.org/10. 1016/j.snb.2015.01.059

13. Sainio S, Palomäki T, Tujunen N, Protopopova V, Koehne J, Kordas $\mathrm{K}$, Koskinen J, Meyyappan M et al (2015) Integrated carbon nanostructures for detection of neurotransmitters. Mol Neurobiol 52:859 866. https://doi.org/10.1007/s12035-015-9233-z

14. Sainio S, Jiang H, Caro MA, Koehne J, Lopez-Acevedo O, Koskinen J, Meyyappan M, Laurila T (2016) Structural morphology of carbon nanofibers grown on different substrates. Carbon 98: 343-351. https://doi.org/10.1016/j.carbon.2015.11.021

15. Venton BJ, Wightman RM (2003) Psychoanalytical electrochemistry: dopamine and behavior. Anal Chem 75:414 A-421 A. https:// doi.org/10.1021/ac031421c

16. Robinson DL, Venton BJ, Heien MLAV, Wightman RM (2003) Detecting subsecond dopamine release with fast-scan cyclic voltammetry in vivo. Clin Chem 49:1763-1773 
17. Laurila T, Sainio S, Jiang H, Koskinen J, Koehne J, Meyyappan M (2016) The role of extra carbon source during the pre-annealing stage in the growth of carbon nanofibers. Carbon N Y 100:351354. https://doi.org/10.1016/j.carbon.2016.01.037

18. Sainio S, Nordlund D, Gandhiraman R, Jiang H, Koehne J, Koskinen J, Meyyappan M, Laurila T (2016) What does nitric acid really do to carbon nanofibers? J Phys Chem C 120:22655-22662. https://doi.org/10.1021/acs.jpcc.6b06353

19. Laurila T, Sainio S, Jiang H, Isoaho N, Koehne JE, Etula J, Koskinen J, Meyyappan M (2017) Application-specific catalyst layers: Pt-containing carbon nanofibers for hydrogen peroxide detection. ACS Omega 2:496-507. https://doi.org/10.1021/ acsomega.6b00441

20. Wester N, Sainio S, Palomäki T, Nordlund D, Singh VK, Johansson LS, Koskinen J, Laurila T (2017) Partially reduced graphene oxide modified tetrahedral amorphous carbon thin-film electrodes as a platform for nanomolar detection of dopamine. J Phys Chem C 121:8153-8164. https://doi.org/10.1021/acs.jpcc.6b13019

21. Rand E, Periyakaruppan A, Tanaka Z, Zhang DA, Marsh MP, Andrews RJ, Lee KH, Chen B et al (2013) A carbon nanofiber based biosensor for simultaneous detection of dopamine and serotonin in the presence of ascorbic acid. Biosens Bioelectron 42:434 438. https://doi.org/10.1016/j.bios.2012.10.080

22. Peltola E, Heikkinen JJ, Sovanto K, Sainio S, Aarva A, Franssila S, Jokinen V, Laurila T (2017) SU-8 based pyrolytic carbon for the electrochemical detection of dopamine. J Mater Chem B Mater Biol Med 5:9033-9044. https://doi.org/10.1039/C7TB02469J

23. Tujunen N, Kaivosoja E, Protopopova V, Valle-Delgado JJ, Österberg M, Koskinen J, Laurila T (2015) Electrochemical detection of hydrogen peroxide on platinum-containing tetrahedral amorphous carbon sensors and evaluation of their biofouling properties. Mater Sci Eng C Mater Biol Appl 55:70-78. https://doi.org/ 10.1016/j.msec.2015.05.060

24. Isoaho N, Peltola E, Sainio S, Wester N, Protopopova V, Wilson BP, Koskinen J, Laurila T (2017) Carbon nanostructure based platform for enzymatic glutamate biosensors. J Phys Chem C 121:46184626. https://doi.org/10.1021/acs.jpcc.6b10612

25. Isoaho N, Wester N, Peltola E, Johansson LS, Boronat A, Koskinen J, Feliu J, Climent V et al (2017) Amorphous carbon thin film electrodes with intrinsic Pt-gradient for hydrogen peroxide detection. Electrochim Acta 251:60-70. https://doi.org/10.1016/j. electacta.2017.08.110

26. Caro MA, Aarva A, Deringer VL, Csányi G, Laurila T (2018) Reactivity of amorphous carbon surfaces: rationalizing the role of structural motifs in functionalization using machine learning. Chem Mater 30:7446-7455. https://doi.org/10.1021/acs.chemmater. $8 \mathrm{~b} 03353$

27. Deringer VL, Caro MA, Jana R, Aarva A, Elliott SR, Laurila T, Csányi G, Pastewka L (2018) Computational surface chemistry of tetrahedral amorphous carbon by combining machine learning and density functional theory. Chem Mater 30:7438-7445. https://doi. org/10.1021/acs.chemmater.8b02410

28. Caro MA, Deringer VL, Koskinen J, Laurila T, Csányi G (2018) Growth mechanism and origin of high $\mathrm{sp}^{\wedge}\{3\}$ content in tetrahedral amorphous carbon. Phys Rev Lett 120:166101. https://doi.org/10. 1103/PhysRevLett.120.166101

Publisher's Note Springer Nature remains neutral with regard to jurisdictional claims in published maps and institutional affiliations. 